\title{
Graphene-Carbon Nanotube Hybrids: Synthesis and Application
}

\author{
Khadije Yousefi* \\ Department of Materials Science and Engineering, School of Engineering, Shiraz University, 71348-51154 Shiraz, Iran \\ Received: 06/08/2020 \\ Accepted: 08/11/2020 \\ Published: 20/03/2021
}

\begin{abstract}
Graphene and CNTs have gained considerable concern and research attention. In addition to preventing the aggregation of these carbon compounds, graphene CNT hybridization would also make full use of the synergistic relationship between graphene and CNT. This chapter discusses the different carbon nanomaterials and their special properties, and a thorough analysis of the graphene-CNT derivatives is observed. It would also discuss in detail the methods and their properties used to create graphene-CNT hybrids. Their applications are also described particularly in device sensing, energy/supercapacitors, and material science.
\end{abstract}

Keywords: Carbon nanotubes, graphene, CNT-graphene hybrid

\section{Introduction}

Completely carbon-based products from activated carbon, carbon nanotubes (CNTs) to graphene have gained widespread prominence due to their intricate nanostructures, physical and chemical characteristics of high quality. Such features involve a range of materials (powders, fabrics, aerogels, composites, boards, monoliths, columns, etc.), extremely inert electrochemistry, fast storage, and porosity regulation (1-3). Graphene and CNTs generated tremendous attention and involvement in science due to their remarkable physical residences, such as extreme electrical conductivity, equal thermal stability, and excellent mechanical efficiency (4). Conversely, due to the powerful vanderWaals forces among them, the agglomeration of carbon materials, especially graphene and CNTs, is unavoidable. Graphene CNT hybridization often does not help to inhibit the association of such carbon compounds, but may also show synergistic results between graphene and $\operatorname{CNT}(5,6)$. Research has shown that hybrid graphene-CNT nanomaterials have more electrical conductivity, superior surface structure, and improved catalytic properties relative to standard CNT or graphene $(6,7)$. The hybrid system is efficient and can be assembled using a range of techniques like simple solution assembly, chemical vapor deposition, and CNT discharge $(8,9)$. This chapter demonstrates and discusses methods for hybridizing CNTs with graphene, characterization, and hybrid alertness.

\section{Carbon Nanotubes}

Carbon nanotubes are visible cylinders with one or two additional graphene layers open or near ends (de-called SWCNT, or MWCNT). The diameters of SWCNT and MWCNT are usually $0.8-2 \mathrm{~nm}$ and 5-20 $\mathrm{nm}$ respectively, whereas the diameters of MWCNT maybe $100 \mathrm{~nm}$, respectively. CNTs have low mass density, immoderate durability, and high issue ratio (usually about 300-1000), leading in mechanical, thermal, and electrical properties that are highly satisfactory(10). Theoretical and experimental studies on individual SWCNT show significantly strong tensile strength (150-180 GPa) and tensile modulus (640 GPa-1 TPA), more powerful than popular carbon fibers (11). Also, they display off enormous thermal and electric properties a number of the great mechanical properties associated with CNTs: thermally stable in vacuum up to $2800^{\circ} \mathrm{C}$, thermal conductivity two times as proper as concrete, electronic present day output 1000 extra times than copper wires (12). CNTs may be single-walled or multi-walled structures, figure 1 shows an image of MWCNT nanostructure transmission electron microscope (TEM), in which several graphical carbon layers and a hollow core appear.

\section{Graphene Nanosheets}

Graphene is a 2D single-atom-thick film of carbon atoms assembled hexagonally $(13,14)$. The carbon bonds are $\mathrm{sp}^{2}$ hybridized where the in-plane $\sigma_{C-C}$ bond is one of the most strong links to the substance and the out-of-plane relation is responsible for the conduction of graphene electron contributing to a delocalized electron network. Graphene has shown remarkable physical properties due to the specific structural qualities which have provided substantial attention to research in both science and technology communities (15-18). Graphene has been used as a building block for many of such carbon allotropes in extraordinary lengths, as visible in Figure.2 (19). For example, with a spacing of $0.33-0.34 \mathrm{~nm}$, 3D graphite is composed of graph nanosheets layered at each other's pinnacle. One-dimensional (1D) carbon allotropes, CNTs which include SWCNTs and MWCNTs can be produced in miles by rolling the graphene into single or multi-walled tubular nanostructures. Wrapping a piece of graphene like a ball often has effects in zero-dimensional (OD) fullerenes.

*Corresponding author: Khadije Yousefi, Department of Materials Science and Engineering, School of Engineering, Shiraz University, 71348-51154 Shiraz, Iran. E-mail: khadije.yousefi@gmail.com 


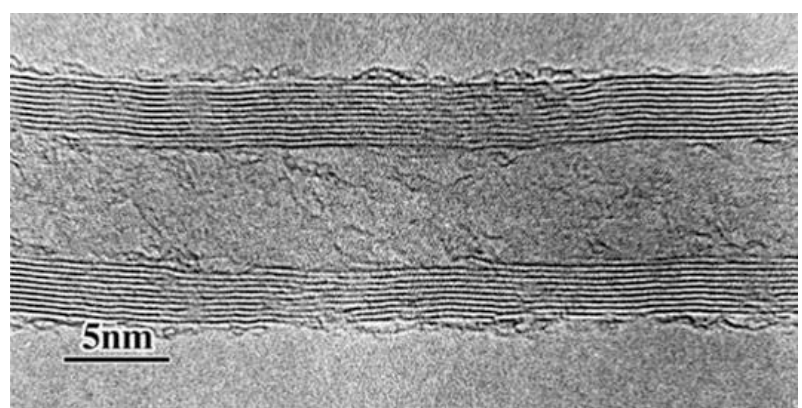

Fig. 1: TEM micrograph demonstrating the layered structure of a carbon nanotube with multiwalls (12)

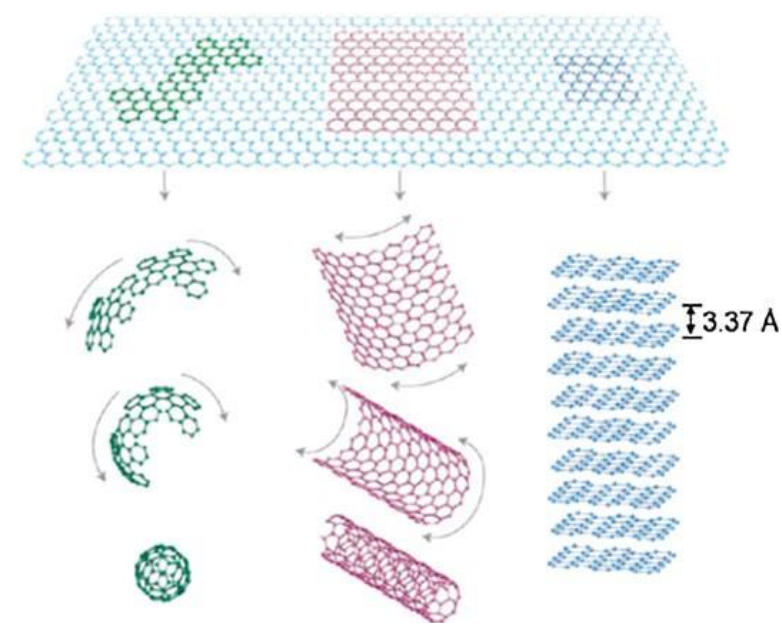

Fig. 2: Graphene, the building block of all graphic types, can be covered in OD buckyballs, formed in 1D nanotubes, and packed in 3D graphite (19)

\section{Strategies for the Hybridization of CNTs with Graphene}

1D CNT and 2D graphene hybridization create nanostructured hybrids with different topological architectures (10). Therefore, the CNT and graphene derivatives are classified into three groups compatible with their different nanostructures: As seen in Fig.3, CNTs are horizontally adsorbed to the base of the graphene, CNTs adsorbed by the graphene floor and CNT covered in particular graphene. Graphene acted as a non-stop layer matrix for CNTs horizontally adsorbed to graphene surfaces to retain the graphene-CNT hybrids, the most common type. For CNTs perpendicularly adsorbed to the graphene surface, this process can be due to a single point of contact between the graphene sheet and CNTs, where CNTs connect graphene sheets during chemical vapor deposition via the catalyst particles (CVD). The method of hybrid wrapping focuses on the contact between graphene sheets and CNTs, as well as the number of CNTs wrapped in graphene nanosheets. Graphene oxide (GO) or functional graphene hybridization with CNTs is usually achieved using different methods. The assembly process practically blends graphene and CNT, while the approach in situ consists of scraping CNTs from graphene sheets into CNT / GNR or CNT types. 4.1 Assembly Method

Because of their aromatic $\mathrm{sp}_{2}$ nanostructure, graphene and CNTs are co-assembled for the assembly of hierarchical structures by van der Waals, or similarities with $\pi-\pi$. Considerably oxygenated mechanical moving surface groups or mechanical CNT Groups(20). Graphene is used for electrostatic or covalent assemblies; GNS graphene nanosheets; graphene oxide reduced by rGO; Acid-dealing with A-MWCNT MWCNT; PPD p-Phenylenediamine; SDBS Sodium dodecylbenzene sulfonate. As illustrated in the figure.4e, GO sheets with several conjugated clusters were likely to interact with MWCNTs when the weight ratio of MWCNT to GO sheets was 2:1, creating exfoliated GO sheets with MWCNT coatings (Fig. 4f). Widespread MWCNT agglomeration - GO complexes have been identified as immoderate MWCNTs and twisted constant tubular CNTs on moving sheets reducing the solubility of MWCNT - transfer complexes. Because the load ratio of MWCNTs to sheets is 1:2 (Fig. 4 g), the single GO sheet will always communicate with loads of MWCNTs at first. Clear suspensions of MWCNT motion complexes were developed after the dynamic equilibrium phase due to the long-term sonication (the inset image in Fig $4 \mathrm{~g}$ ). A small number of hair-like MWCNTs are naturally fixed at the surface of GO sheets (Fig. 4h), raising the hydrophilic MWCNT - GO complexes (21).

Liu et al. $(22,23)$ stated that the hybrid graphene oxide nanoribbon/carbon nanotube (GONR / CNT) (Fig. 2.17a) has become an easy method for unzipping. The left MWCNTs will link to equipped-made GNRs to form interconnected 3-D carbon networks with partially unwrapped MWCNTs. After being chemically modified using hydrazine hydrate $(65 \mathrm{~S}$ $\mathrm{cm}-1$ ), GNR / CNT hybrids can be produced with an advanced conductivity of $120 \mathrm{~S} \mathrm{~cm}-1$ compared to that of CNTs(22). As seen in Fig. 5c similar weight ratios of GONRs in GONR / CNT mixtures may be conveniently prepared and measured using XRD styles where the GONR peak $\left(2=11.28^{\circ}\right)$ is reduced because the CNT peak $\left(2=26.18^{\circ}\right)$ reduced.

\section{Type 1}

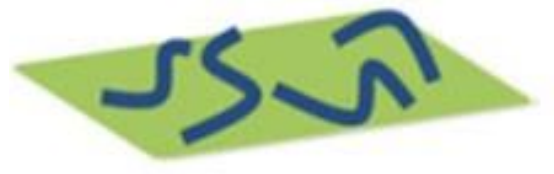

Type 2

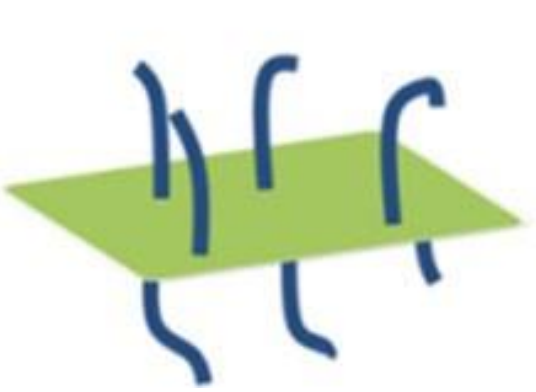

Fig. 3: Three types of the hybrids of graphene and CNTs (9)
Type 3

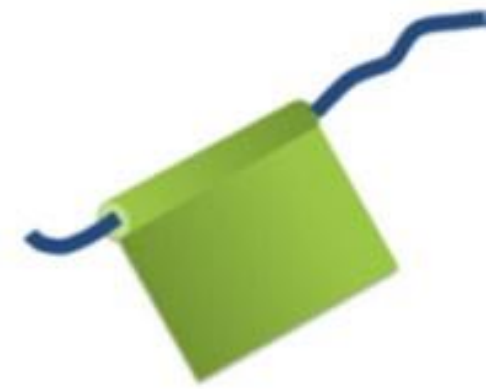


The significance of their function peaks and can deliver asorganized GONR / CNT derivatives to the GONR weight ratios as 16,55 and 85 percent respectively can be derived from a relationship curve. They were described for simplicity as GONR16 in step with cent / CNT, GONR55 in keeping with cent / CNT, and GONR85 in keeping with cent / CNT. The GNR / CNT combination TEM and SEM stats (Fig. 6) with different GNR weight ratios indicate that within the hybrids GNRs are aligned with CNTs and independent GNRs are simply proved as the unzipping diploma decreases.

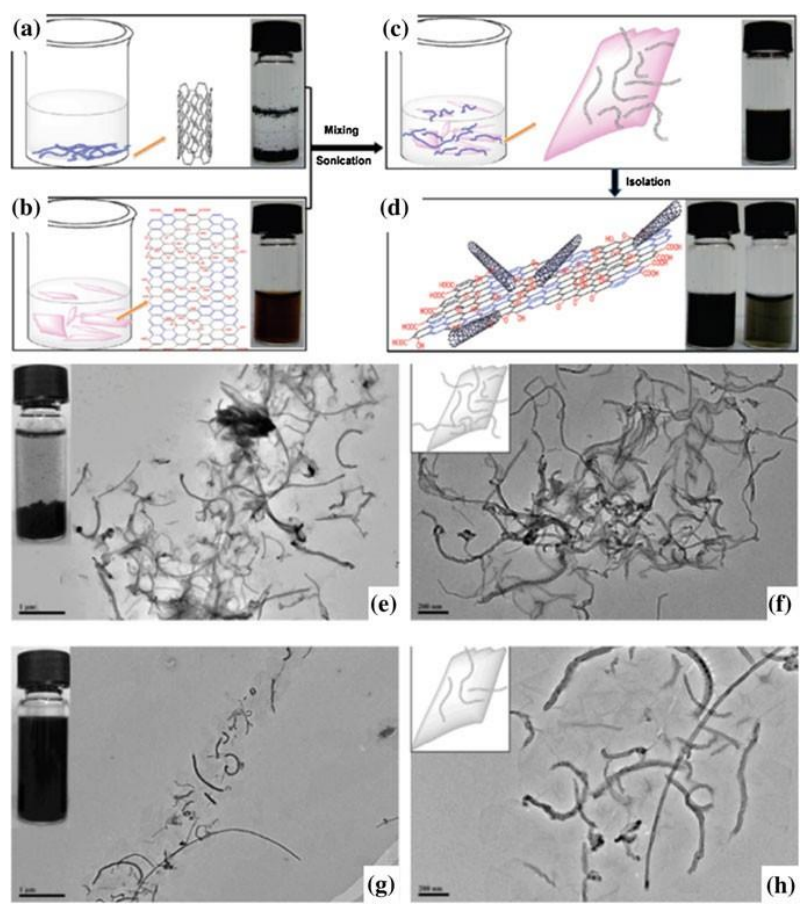

Fig. 4 a-d: A schematic for the formation of the GO-MWCNT hybrids. TEM images of GO- MWCNT hybrids by adjusting the initial weight ratio of MWCNTs to GO sheets with 2:1 e, fand 1:2 g, h at low and high magnifications (21)

\subsection{In Situ Method}

The results of assembly methods are usually less specific and this slowly influences the conductivity and surface region, since this approach is less complex than in situ development. Hybrid growth in situ graphene-CNT is more complex in evaluation, but it maintains an extra impact on product content and sensitivity to form the density, and hybrid shape. The development of the graphene structures CNT-pillared go and CNT-pillared was established (Fig. 5) (24). The cross-sectional images are taken from the sample display nanostructures of layers of CNTs as nanopillars among the move and graphene surfaces. extended CNTs may be produced using growing the quantity of the Ni catalyst while shorter CNTs may be obtained by way of elevating the growth duration for CVDs. CNTpillared rGO composite substances display exceptionally strong visible light photocatalytic efficiency in decaying dye Rhodamine $\mathrm{B}$, owing to the exceptional porous nature and impressive electron transfer properties of graphene.

\section{Application of Graphene-Carbon Nanotube Hybrids}

Carbon nanotube-graphene hybrid possesses the outstanding capacity for a number uses $(25,26)$. In this chapter, we'll describe the applications of this amazing CNT-graphene hybrid tool.

\subsection{Sensors}

The developing variety of diabetic sufferers round the sector has sparked interest in medical biosensing, and in that regard, the supremacy of glucose biosensors. The biosensor detects enzymes usually through amperometry, although the latest instances of non-enzymatic detection of glucose have been recorded. For instance, GO electrode $(27,28)$ and Pt nanoflowers covered on the in situ CNT-graphene hybrid (29) have been utilized in non-enzymatic blood glucose sensing. For non-enzymatic blood glucose sensing hybrid CNT-graphene (29) was observed. As described, the enzymatic glucose biosensors use glucose oxidase (GOx) due to its balance and specificity to glucose. The chemistry for this technique may be contained under literature(30). The enzyme Flavin adenine nucleotide (FAD) is at the energetic center of the redox reaction(30). Due to enzymatic action, the electrode-generated $\mathrm{H}_{2} \mathrm{O}_{2}$ has been transformed into electrochemically detected, starting with designed glucose biosensors, although the production of $\mathrm{H}_{2} \mathrm{O}_{2}$ is based on oxygen, requiring excessive detection potential which interferes with other redox composites. In second-generation biosensors, but, oxygen dependency is eliminated way to redox mediators. Such mediators are in continuous movement and can be connected to an enzyme that movements electrons to the electrode or diffuse without FAD impediments or spherical the other manner, ensuing in amperometric signal manufacturing. Glucose biosensing in third-generation biosensors can be achieved at a lower capability without the want for redox mediators via direct electron transfer (DET) from the redox core to the electro. Table 1 summarizes the efficiency of various recorded graphene hybrids in totally glucose-based biosensing DET.

Direct electron transfer with bare electrodes of GOx enzyme is difficult to accomplish because FAD is deeply embedded inside the structure. For robust electron transfer kinetics and enzyme immobilization to mediator-loose DET for GOx, CNT-graphene hybridized shape provides a distinctly conducive and methodical matrix with superb effects on glucose sensing. While the exact role of DET remains unclear, the advanced electrochemical properties of the extremely conductive graphene and CNT-graphene hybrid contiguous graphene provide treasured guidelines. The lifestyles of FAD redox tops in cyclic voltammetry with average potential approximating the usual electrode capacity of GOx are a huge indicator of the GOx DET scheme (31). Glucose is then measured by calculating the increase in the top contemporary of certainly charged FAD in glucose oxidation reaction or the decrease in the peak current of the negatively charged FAD due to the use/decline of oxygen. Glucose evaluation the usage of oxygen may be carried out at low capability as an example of mediator-loose sensing and hence extraordinary of sensors of the third generation. CNT-graphene hybrid offers enzyme immobilization with an extended floor position and produces 3-d conductive matrix for efficient electron transfers to neighboring moleculesGOx is immobilized from the redox enzyme motion center at the CNT-graphene electrode and DET in the course of the oxidation mechanism as shown in figure 7. It has been shown to a great extent that CNT-graphene hybrid, built using physical means using CNTs, achieves DET for GOx through cathode-based oxygen-biosensing glucose $(32,33)$. 
(a)
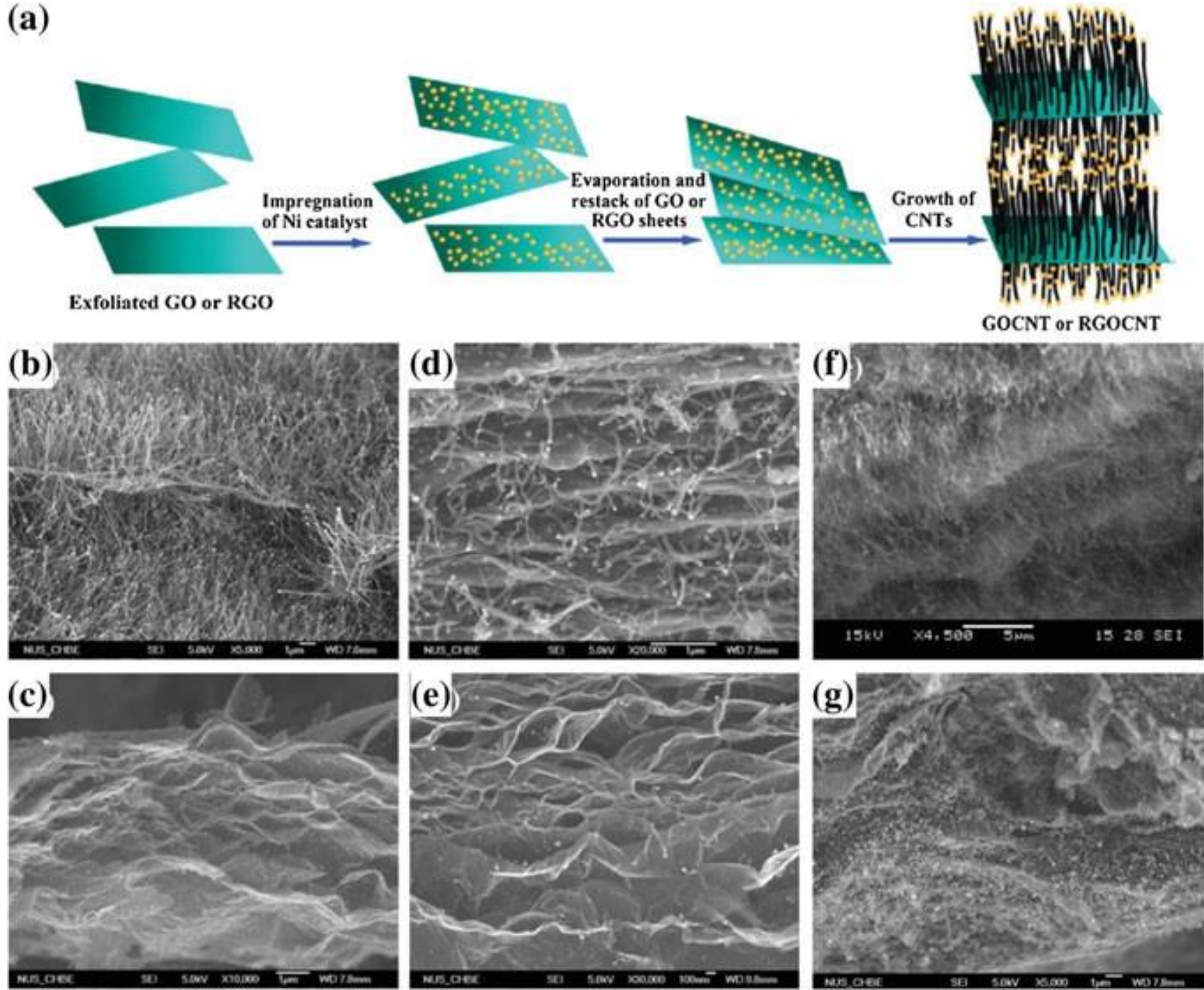

Fig 5: Experimental methods pillar the go and graphene platelets using MWCNTs. B-GOCNT-30-17, c GOCNT-15-17, $d$ GOCNT-30-9 and e GOCNT15-9, $f$ RGOCNT-15-4 and g, respectively, RGOCNT-15-0.6. The final samples are named, respectively, for the CNT-pillared go and RGO composites as GOCNT-X-Y or RGOCNT-X-Y.X illustrates here the CVD period (15 or $30 \mathrm{~min}$ ) if Y refers to the catalyst of Ni / C mass loading (24)

Also, the electrochemically lively space for CNT-graphene hybrid altered with $\mathrm{ZnO}$ nanoparticles has been multiplied, increasing the possibility of stepped-up enzyme immobilization and rapid electron switching (34). This technique employs differential pulse voltammetry to come across glucose taking benefit of oxygen depletion inside the system.

\subsection{Sensitized Solar Cells}

After Grätzel 's institution published nanostructured $\mathrm{TiO}_{2}$ films on anode electrodes in 1991 (35), sensitized solar cells (SSCs) attracted considerable interest in the photoelectric conversion of solar energy due to their fantastically low value and incredible efficiency. SSCs are photoelectrochemical structures with an electrolyte, a counter electrode, and a photoanode; The photoanode is typically a semiconductor, often $\mathrm{ZnO}$ and $\mathrm{TiO}_{2}$, sensitized to photoactive materials such as colorants (DSSC), biomolecules (BSSC), and quantum dots (QSSC), mounted on a transparent conductive surface which transfers photogenerated electrons from the semiconductor to the outer circuit. Graphene converted into transferred in SSC to both photoanode and counter electrode, serving excellent positions. This can also be used as a clear conductive film in the photoanode. As a conductive film, it can improve widely used metal oxides, such as FTO and ITO, as it overcomes the disadvantages of steel oxides, such because size, robustness, chemical, and mechanical power and improved near-infrared transmission $(28,36)$. It can also act as an additive to the semiconductor surface for electrical bridging. Secondly, graphene quantum dots can be used as the sensitizer has semiconductor residences such that photons excite electrons from the HOMO to his LUMO. Ultimately, graphene will be part of the counter-electrode, improve electron transport, and enhance the redox attraction. Table 2 summarizes the average performance, recorded in literature, of different types of SSCs based on graphene derivatives. 


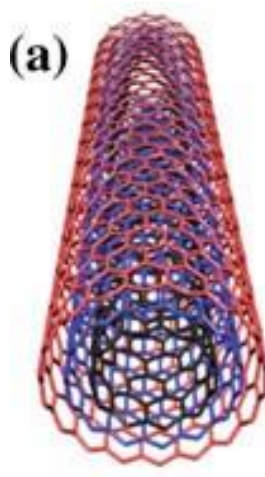

CNTs

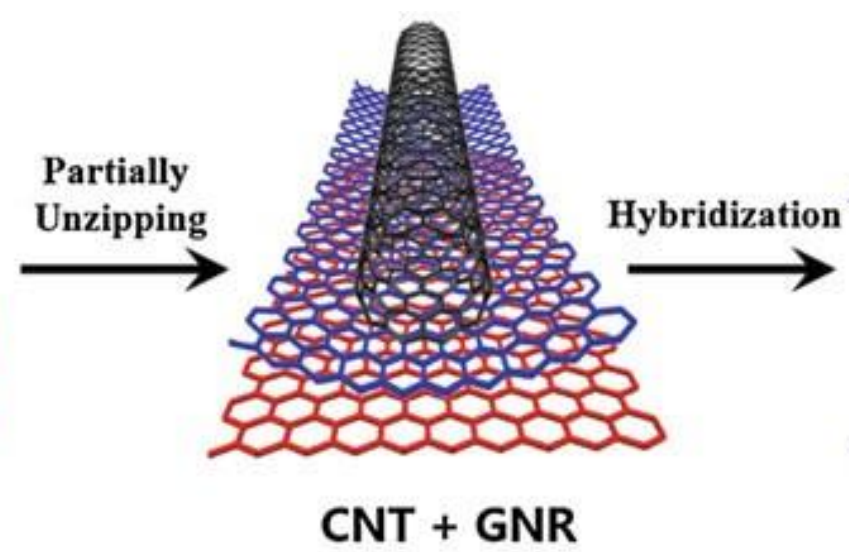

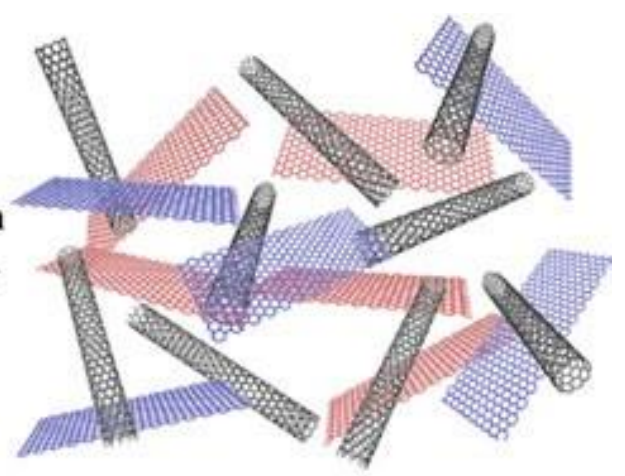

CNT-GNR hybrids
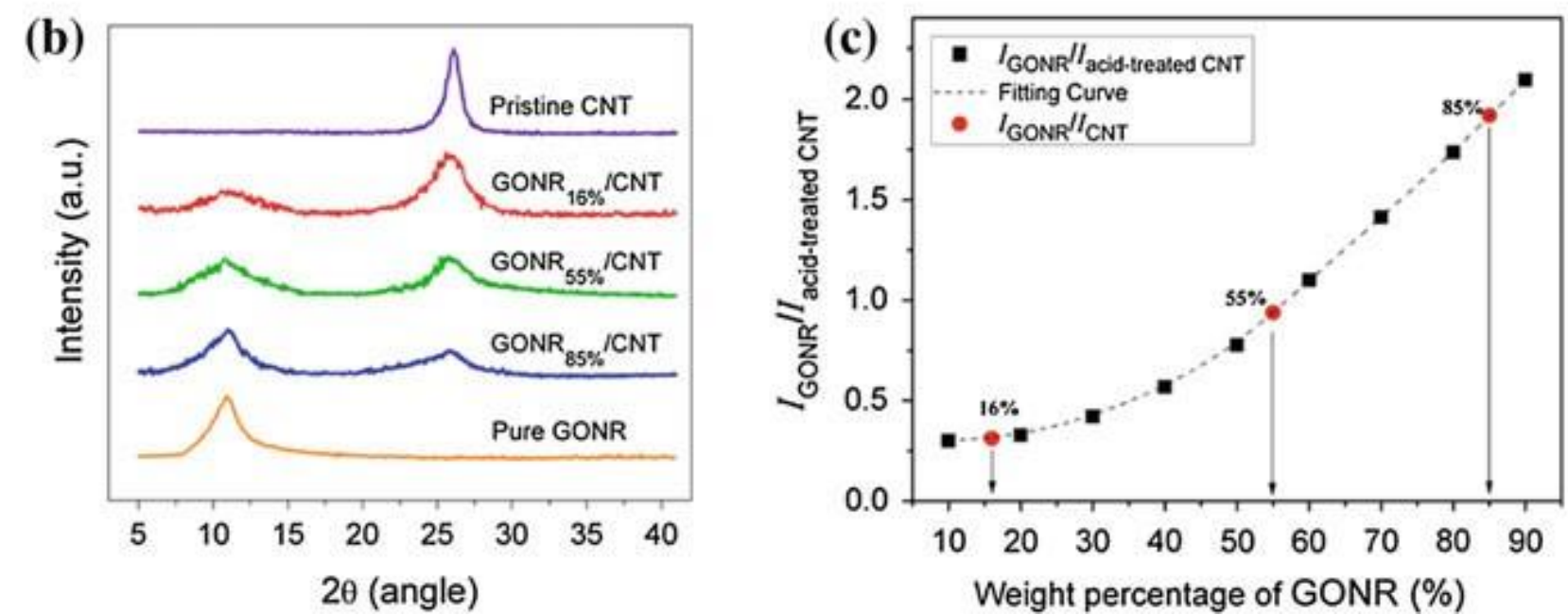

Fig. 6: A GNR / CNT mixed production flow model. B XRD clean CNT architectures, GONR / CNT mixture with separate weight ratios and GONR simple architecture. C GONR weight ratio estimates in combinations GONR / CNT are based on GONR weight percentage and power ratio of characteristic peaks in their XRD shape (22)

Table 1: Summary of performances of graphene hybrids in DET based glucose biosensors

\begin{tabular}{|c|c|c|c|c|c|c|}
\hline Type of Hybrid & Electrode & $\mathrm{Ks}\left(\mathrm{s}^{-1}\right)$ & $\begin{array}{l}\text { Detection } \\
\text { range }(\mathrm{mM})\end{array}$ & Sensitivity $\left(\mu \mathrm{AmM}^{-1} \mathrm{~cm}^{-2}\right.$ & $\begin{array}{l}\text { LOD } \\
(\mu \mathrm{M})\end{array}$ & Refer \\
\hline \multirow[t]{3}{*}{ Graphene-CNT } & $\begin{array}{l}\text { GOx/electrochemically } \\
\text { reduced G- } \\
\text { MWCNT/GCE with } \\
\text { FMCA }\end{array}$ & 3.02 & $0.01-6.5$ & ( & 4.7 & (37) \\
\hline & $\begin{array}{l}\text { GOx/chemically } \\
\text { reduced G-CNT/GCE }\end{array}$ & $\begin{array}{l}\text { Not } \\
\text { reported }\end{array}$ & Up to 8 & 1.27 & $\begin{array}{l}\text { Not } \\
\text { reported }\end{array}$ & (38) \\
\hline & GOx/MWCNT/GO & 11.22 & $0.1-19.82$ & 0.266 & 28 & $(32)$ \\
\hline \multirow{3}{*}{$\begin{array}{l}\text { Graphene-metal } \\
\text { nanoparticle }\end{array}$} & $\begin{array}{l}\text { GOx-AuNP-chitosan- } \\
\text { graphene nanosheets }\end{array}$ & $\begin{array}{l}\text { Not } \\
\text { reported }\end{array}$ & $0.0021-0.0057$ & 79.71 & 0.7 & (39) \\
\hline & $\begin{array}{l}\text { GOx/PAMAM } \\
\text { dendrimer/AgNP/RGO }\end{array}$ & 8.59 & $0.032-1.89$ & 75.72 & 4.5 & $(40)$ \\
\hline & $\begin{array}{l}\text { GOx/AuNP/bilayer } \\
\text { graphene }\end{array}$ & $\begin{array}{l}7.74 \pm \\
0.16\end{array}$ & $0.1-10$ & $\begin{array}{l}\text { For Human serum:64 } \mu \mathrm{A} \\
\mathrm{mM}^{-}\end{array}$ & 35 & (41) \\
\hline \multirow{3}{*}{ Graphene-SNMs } & GOx/CdS anoparticles & $\begin{array}{l}\text { Not } \\
\text { reported }\end{array}$ & $0.05-11$ & 7 & 50 & (39) \\
\hline & GOx/CdS nanocrystals & 5.9 & $2-16$ & 1.76 & 700 & (42) \\
\hline & GOx/PLL/RGO/ZrO2 & $\begin{array}{l}5.03 \pm \\
0.14\end{array}$ & $0.29-14$ & $11.65 \pm 0.17$ & $130 \pm 21$ & (23) \\
\hline
\end{tabular}




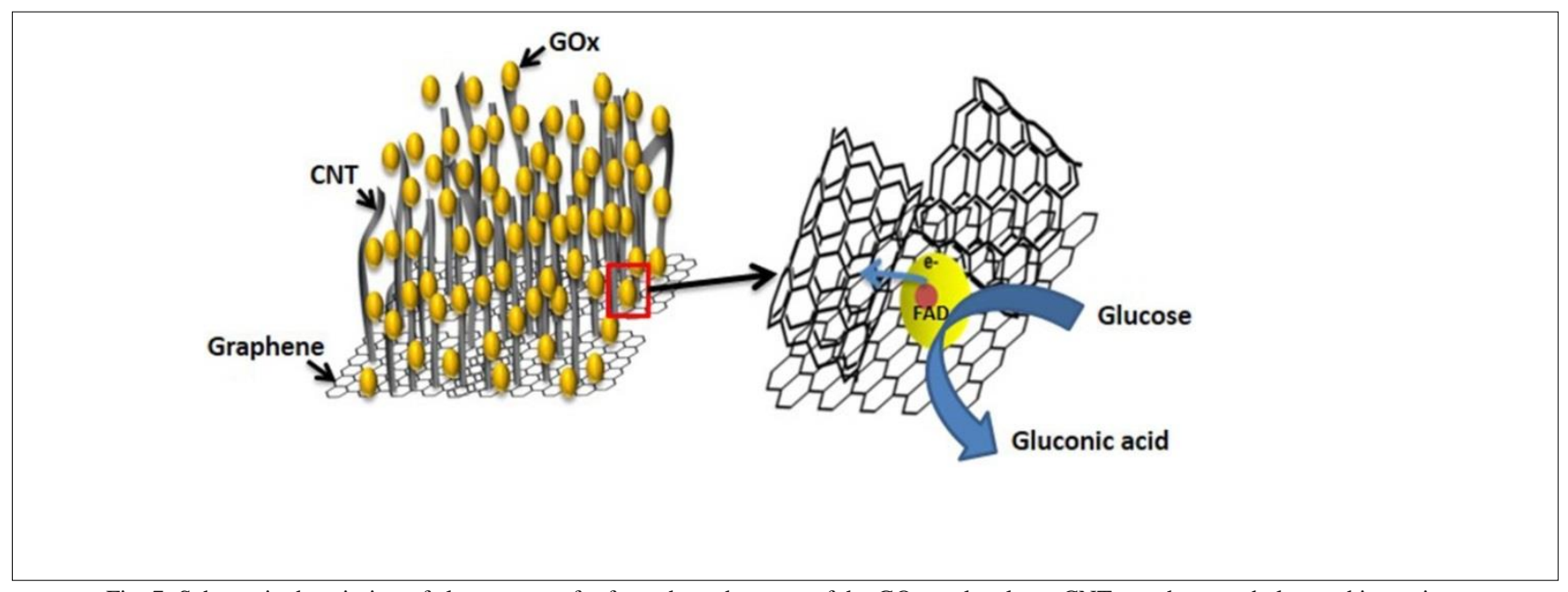

Fig. 7: Schematic description of electron transfer from the redox core of the GOx molecule on CNT-graphene and glucose biosensing

Table 2: Graphene hybrids are used in photochemical/optical sensors

\begin{tabular}{|l|l|l|l|l|l|}
\hline $\begin{array}{l}\text { Electrode with } \\
\text { graphene/SNM hybrid }\end{array}$ & $J_{\mathrm{sc}}\left(\mathrm{mA} / \mathrm{cm}^{2}\right)$ & $V \mathrm{oc}(\mathrm{V})$ & $F F(\%)$ & $n(\%)$ & References \\
\hline CE: rGO-CNT/FTO & 15.25 & 0.68 & 51.05 & 5.29 & $(43)$ \\
\hline CE: VACNT-graphene paper & 14.24 & 0.68 & 62.4 & 6.05 & $(44)$ \\
\hline PA: MWCNT-rGO-TiO2/FTO & 11.27 & 0.78 & 70 & 6.11 & $(45)$ \\
\hline CE: TiN-rGO-CNT/FTO & 14.0 & 0.642 & 46 & 4.13 & $(46)$ \\
\hline CE: CuInS2-rGO/FTO & 16.63 & 0.74 & 51 & 6.18 & $(47)$ \\
\hline PA: Graphene QD/TiO2/FTO & 0.2 & 0.48 & 58 & NA & $(48)$ \\
\hline PA: N3/TiO2-rGO/ & 16.29 & 0.69 & NA & 6.97 & $(49)$ \\
\hline CE: CNT-rGO/graphite paper & 12.86 & 0.78 & 61.3 & 6.17 & $(50)$ \\
\hline
\end{tabular}

\subsubsection{Graphene-CNT hybrids in SSCs}

Graphene-CNT hybrid compounds have been evaluated for use in SSC mainly as a counter-electrode, but also as part of the electrolyte and photoanode (51). RGO-CNT was integrated into the counter electrode and noticed that CNT flake gaps and increased electrical conductivity were bridged. The hybrid's strong catalytic and electrical properties enabled the acquisition of portable output, which was barely reduced but comparable to the usage of a Pt counter electrode, taking into account the cost benefits and mechanical versatility of carbon nanomaterials. By partially unzipping MWCNT, Zhibin et.al (52) recorded better graphene nanoribbons- CNT hybrid performance compared to $\mathrm{Pt}$, which resulted in CNT being bridged with very wide floor location. Another hybrid built with VACNT was developed on $\mathrm{Li}$ et .al(44) converted graphene paper. In advanced SSC counter-electrode allowing cellular production to hit $83 \%$ of that with a Pt film electrode and better-tangled CNT efficiency due to shorter transport routes. Ming-Yu et al. (45) documented the benefits of MWCNT and graphene mixing in photoanode, where MWCNT adsorption confirmed a decrease in the recombination and deposition of graphene sheets and improved the degree of adsorption of dye. Although the combination of graphene and CNT with ionic beverages in a quasi-solid electrolyte. Kingdom has shown that Ahmad et al. (53) has significantly increased the energy conversion performance of cell phones from 0.16 to 2.5 percent because carbon nanomaterials serve as charging transporters in ionic liquids and as catalysts for electrochemical $\mathrm{I}^{3-}$ reduction.

\subsection{Supercapacitors}

The supercapacitors have considerably higher power densities than traditional dielectric condensers and demonstrate quick charging and discharge rates, long cycle life, and inadequate energy densities for batteries and fuel cells.

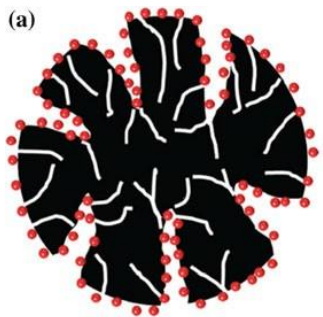

(c)

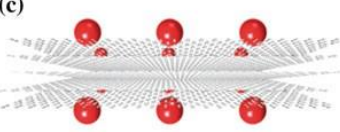

(b)

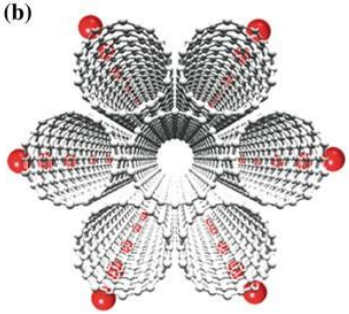

(d)

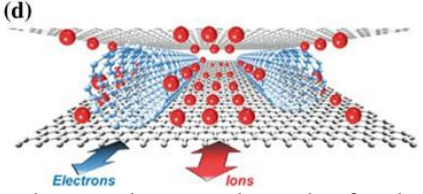

Fig. 8: Assessment of different carbon products as electrodes for the supercapacitor. (a) an Activated carbon. Activated carbon has a very large surface area. Nevertheless, Electrolyte ions can not reach many of the micropores. (b) CNT bundles. Usually, CNTs form bundles which limit their surface area. Electrolyte ions may only reach the outermost surface. (c) Pristine graphene. During the drying cycle, graphene nanosheets are likely to agglomerate through van der Waals interactions. Electrolyte ions would have trouble accessing ultrasmall pores, especially for larger ions such as an organic electrolyte or at a high charge rate. (d) Graphene-CNT composite. CNTs can serve as a spacer between the nanosheets of graphene to provide the electrolyte ions with rapid diffusion pathways. Electrical conduction may be improved for the electrons. The CNTs often act as a binder to tie the nanosheets intact to avoid the disintegration of the graphene structure of graphene in electrolyte $(4,54)$. 
(a)
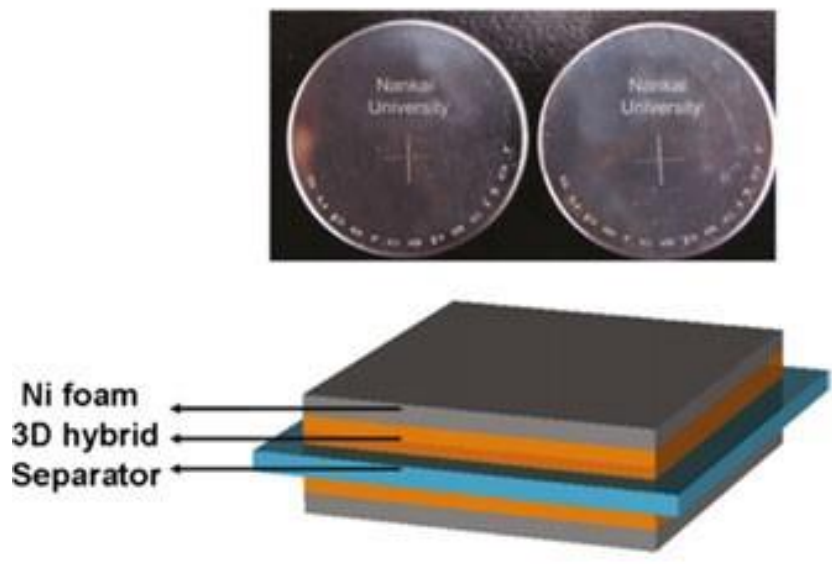

(c)

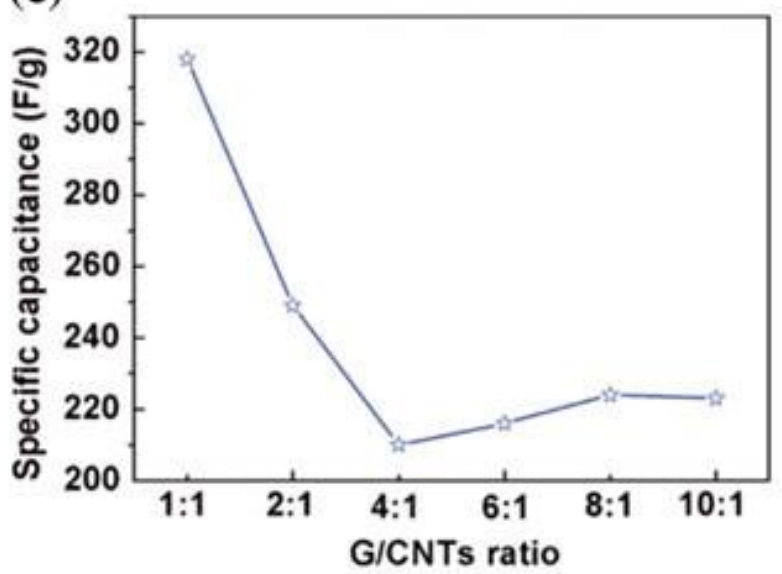

(b)

$\begin{array}{cccccc}\text { G:CNT ratio } & C_{1}, \mathrm{~F} / \mathrm{g} & \begin{array}{c}E_{\mathrm{b}} \\ (\mathrm{Wh}) / \mathrm{kg}\end{array} & C_{\mathrm{O}} \mathrm{F} / \mathrm{g} & \begin{array}{c}E_{\mathrm{G}} \\ (\mathrm{W} \mathrm{h}) / \mathrm{kg}\end{array} & \begin{array}{c}\text { BET SSA, } \\ \mathrm{m}^{2} / \mathrm{g}\end{array} \\ 0: 1 & 9 & 0.3 & & & 200 \\ 1: 1 & 112 & 3.9 & 318 & 11.1 & 237 \\ 2: 1 & 129 & 4.5 & 249 & 8.6 & 278 \\ 4: 1 & 143 & 5.0 & 210 & 7.3 & 305 \\ 6: 1 & 164 & 5.7 & 216 & 7.5 & 356 \\ 8: 1 & 181 & 6.6 & 224 & 7.8 & 397 \\ 10: 1 & 187 & 6.5 & 223 & 7.7 & 407 \\ 1: 0 & 190 & 6.6 & 190 & 6.6 & 374\end{array}$

${ }^{a} \mathrm{C}_{\mathrm{t}}$ is the integrated specific capacitance based on the overall weight of the graphene and CNTs, $E_{1}$ is the energy density based on all the weight of both graphene and CNTs, $C_{G}$ and $E_{G}$ are the calculated effective specific capacitance and energy density of the graphene, respectively, and SSA is the specific surface area of the 3-D hybrids.
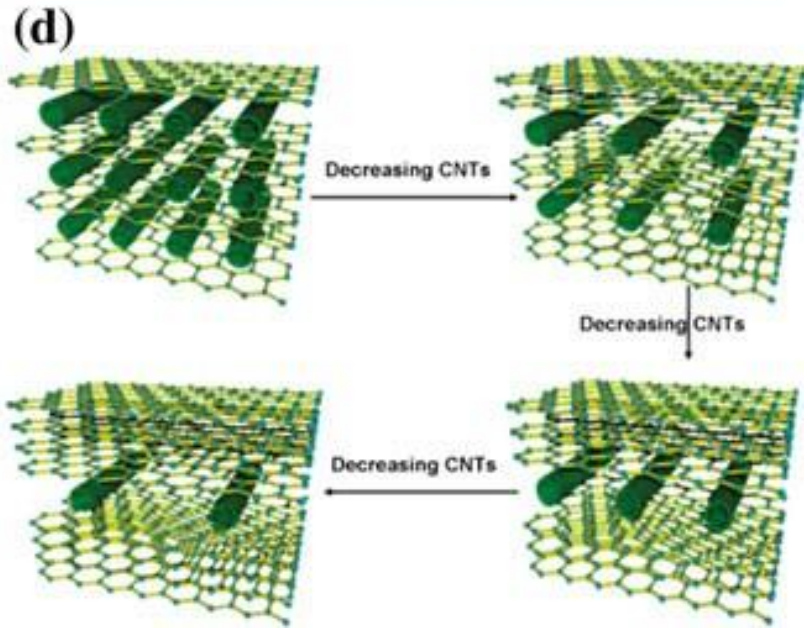

Fig. 9: Supercapacitor architecture basing mainly on the 3-d G / CNT combination. BG / CNT supercapacitor output hybrid, with weight ratios of oneof-a-kind. C strong special graphene capacitance of various G to CNT ratios in hybrid materials. D Schematic diagram of 3-Component hybrid device configuration with CNT load spacer (55)

Owing to their extraordinary physical and chemical properties, carbon-based materials such as activated carbon, CNTs, and graphene were commonly used in supercapacitors with double-layer electrochemical. Different carbon products as electrodes for the supercapacitor have shown in figure 8. By bridging the graphene sheets and decorating a typical capacitive performance, the CNTs will significantly reduce the electrode's electrical resistance to the interlayer. Also, the CNTs can serve as a graphene interlayer spacer to hold away from restacking relative to each individual, allowing available electrolyte ions. The electrolyte ions can readily penetrate the inner portion of the electrodes with the spacer device, and thus greater electrochemically active products can be used (Fig. 8d). However, CNTs may also be used as binders to connect the graphene sheets and to facilitate the adhesion of electrodes to the modern collector (54). The graphene-CNT hybrid-based materials are also particularly suitable for processing electrode items in EDLCs. Chen et al developed and synthesized a hierarchical graphene $(\mathrm{G})$ 3D structure using CNTs due to a transparent and green hydrothermal route between the graphene sheets. The materials of the $3 \mathrm{D}$ hierarchical structure were then used for the production of supercapacitor devices as active electrode materials (Fig. 9a), and high potential strength of 318 $\mathrm{F}$ g-1 was converted into graphene-weight materials with an energy density of $11.1 \mathrm{~W} \mathrm{~h} \mathrm{kg-1}$. The influence of graphene on supercapacitor performance to weight ratio CNT has been studied closely (Fig. 9b, c). First, the maximum overall graphene output is derived from the 1:1 ratio of the G / CNT mixture, providing an unacceptable $318 \mathrm{~F} \mathrm{~g}-1$ power, which is 64 percent of the theoretical value of the graphene. Indeed, the green mechanical power of graphene declines and then rises with the strain ratio of G to CNTs. The schematic image in Fig. $9 \mathrm{~d}$ shows that the restacking of graphene will deteriorate as the ratio of $\mathrm{G}$ to CNTs decreases. It may have a horrible influence on the graphene fabric's effective surface area, thus reducing graphene's effective contribution to the overall capacitance of the entire electrode traveling fabric (G / CNT hybrid).

\section{4 material science}

Recently, Ahmad et al.(56) report exciting applications of CNT-graphene reinforced ceramics in biomedical, aerospace, automotive, and photonic technologies as a result of more suitable longevity and multiple beneficial residences for hybrid starting fabrics. In addition to the $\mathrm{Al}_{2} \mathrm{O}_{3}$ improved output, the considerably better resistance strength of the hybrids may be desirable for a variety of automotive sliding and wearing applications, such as piston rings, cylinder liners, and valve seats $(18,57,58)$. Additionally, the $\mathrm{SiC}, \mathrm{BaTiO} 3$, and Si3N4 impregnated CNT systems were found to depend on properties appropriate for structural use. The chemical and thermally 
stable ceramic hybrids will modify their extreme thermal conductivity, making them appealing for high-temperature applications(59). CNTs / graphene mixture is capable of turning ceramics into usable substances for automotive and aerospace applications. However, CNT-graphene hybrid outof-aircraft electrical properties render $\mathrm{Al}_{2} \mathrm{O}_{3}$.

\section{Conclusion}

The hybrid system is complex and can be designed by many methods including simple solution assembly and process of chemical vapor deposition. The chapter outlines and explores methods for the hybridization of CNTs with graphene and hybrid characterization. This also introduces potential implementations of CNT-graphene hybrid architectures. CNTgraphene has demonstrated superior efficiency in most cases than individual pristine CNTs or graphene, indicating their ability for numerous functional and research applications. Whether as an electrode in mediator-free glucose biosensors, capacitor collector device, electron transfer help, or photoactive material, the usage and work understanding of CNT-graphene hybrid has gradually grown and impacted modern-day carbonbased electronics.

\section{Reference}

1. Chowdhury ZZ, Sagadevan S, Johan RB, Shah ST, Adebesi A, Md $\mathrm{SI}$, et al. A review on electrochemically modified carbon nanotubes (CNTs) membrane for desalination and purification of water. Materials Research Express. 2018;5(10):102001

2. Hashemi SA, Mousavi SM, Arjmand M, Yan N, Sundararaj U. Electrified single-walled carbon nanotube/epoxy nanocomposite via vacuum shock technique: Effect of alignment on electrical conductivity and electromagnetic interference shielding. Polymer Composites. 2018;39(S2):E1139-E48

3. Abdollahifar A, Hashemi SA, Mousavi SM, Rahsepar M. Electromagnetic interference shielding effectiveness of reinforced composite with graphene oxide-lead oxide hybrid nanosheets. Radiation Effects and Defects in Solids. 2019;174(9-10):885-98.

4. Mousavi SM, Hashemi SA, Arjmand M, Amani AM, Sharif F, Jahandideh S. Octadecyl amine functionalized Graphene oxide towards hydrophobic chemical resistant epoxy Nanocomposites. ChemistrySelect. 2018;3(25):7200-7.

5. Kinloch IA, Suhr J, Lou J, Young RJ, Ajayan PM. Composites with carbon nanotubes and graphene: An outlook. Science. 2018;362(6414):547-53.

6. Qiu L, Yang X, Gou X, Yang W, Ma ZF, Wallace GG, et al. Dispersing carbon nanotubes with graphene oxide in water and synergistic effects between graphene derivatives. Chemistry-A European Journal. 2010;16(35):10653-8.

7. Yousefi k, Daneshmanesh H, A. khalife. Optimization of physical and mechanical properties of calcium silicate nanocomposite by Taguchi method. Scientific Journal Management System. 2020;10(39):77-90.

8. Nguyen DD, Cao TT, Le PH, Phan NM. Recent trends in preparation and application of carbon nanotube-graphene hybrid thin films. Advances in Natural Sciences: Nanoscience and Nanotechnology. 2016;7(3):033002.

9. Fan W, Zhang L, Liu T. Graphene-carbon nanotube hybrids for energy and environmental applications: Springer; 2017.

10. Fan S, Chapline MG, Franklin NR, Tombler TW, Cassell AM, Dai $\mathrm{H}$. Self-oriented regular arrays of carbon nanotubes and their field emission properties. Science. 1999;283(5401):512-4.

11. Tasis D, Tagmatarchis N, Bianco A, Prato M. Chemistry of carbon nanotubes. Chemical reviews. 2006;106(3):1105-36.

12. Thostenson ET, Ren Z, Chou T-W. Advances in the science and technology of carbon nanotubes and their composites: a review. Composites science and technology. 2001;61(13):1899-912.

13. Geim AK. Graphene: status and prospects. science. 2009;324(5934):1530-4.

14. Mousavi SM, Hashemi SA, Ghasemi Y. DECORATED GRAPHENE OXIDE WITH OCTADECYL
AMINE/SASOBIT/EPOXY NANOCOMPOSITE VIA VACUUM SHOCK TECHNIQUE: MORPHOLOGY AND BEAMS SHIELDING. Journal of Chemical Technology \& Metallurgy. 2019;54(6).

15. Rao CeNeR, Sood AeK, Subrahmanyam KeS, Govindaraj A. Graphene: the new two-dimensional nanomaterial. Angewandte Chemie International Edition. 2009;48(42):7752-77.

16. Allen MJ, Tung VC, Kaner RB. Honeycomb carbon: a review of graphene. Chemical reviews. 2010;110(1):132-45.

17. Sun Y, Wu Q, Shi G. Graphene based new energy materials. Energy \& Environmental Science. 2011;4(4):1113-32.

18. Mousavi SM, Hashemi SA, Ghasemi Y, Amani AM, Babapoor A Arjmand O. Applications of graphene oxide in case of nanomedicines and nanocarriers for biomolecules: review study. Drug metabolism reviews. 2019;51(1):12-41

19. Geim AK, Novoselov KS. The rise of graphene. Nanoscience and technology: a collection of reviews from nature journals: World Scientific; 2010. p. 11-9.

20. Khan U, O'Connor I, Gun'ko YK, Coleman JN. The preparation of hybrid films of carbon nanotubes and nano-graphite/graphene with excellent mechanical and electrical properties. Carbon. 2010;48(10):2825-30.

21. Zhang C, Ren L, Wang X, Liu T. Graphene oxide-assisted dispersion of pristine multiwalled carbon nanotubes in aqueous media. The Journal of Physical Chemistry C. 2010;114(26):1143540.

22. Yang Z, Liu M, Zhang C, Tjiu WW, Liu T, Peng H. Carbon nanotubes bridged with graphene nanoribbons and their use in high-efficiency dye-sensitized solar cells. Angewandte Chemie. 2013;125(14):4088-91.

23. Liu M, Miao Y-E, Zhang C, Tjiu WW, Yang Z, Peng H, et al Hierarchical composites of polyaniline-graphene nanoribbonscarbon nanotubes as electrode materials in all-solid-state supercapacitors. Nanoscale. 2013;5(16):7312-20.

24. Zhang LL, Xiong Z, Zhao X. Pillaring chemically exfoliated graphene oxide with carbon nanotubes for photocatalytic degradation of dyes under visible light irradiation. Acs Nano. 2010;4(11):7030-6.

25. Koay H, Ruslinda AR, Hashwan SSB, Fatin M, Thivina V, Tony VS, et al., editors. Surface morphology of reduced graphene oxidecarbon nanotubes hybrid film for bio-sensing applications. 2016 IEEE International Conference on Semiconductor Electronics (ICSE); 2016: IEEE.

26. Ding M. Hybrid Materials Based on Carbon Nanotubes and Graphene: Synthesis, Interfacial Processes, and Applications in Chemical Sensing: University of Pittsburgh; 2013.

27. Wu G-h, Song X-h, Wu Y-F, Chen X-m, Luo F, Chen X. Nonenzymatic electrochemical glucose sensor based on platinum nanoflowers supported on graphene oxide. Talanta. 2013;105:379 85 .

28. Abdollahifar A, Hashemi SA, Mousavi SM, Rahsepar M, Amani AM. Fabrication of graphene oxide-lead oxide epoxy based composite with enhanced chemical resistance, hydrophobicity and thermo-mechanical properties. Advances in Polymer Technology. 2018;37(8):3792-803.

29. Badhulika S, Paul RK, Terse T, Mulchandani A. Nonenzymatic glucose sensor based on platinum nanoflowers decorated multiwalled carbon nanotubes-graphene hybrid electrode. Electroanalysis. 2014;26(1):103-8.

30. Ijeomah G, Obite F, Rahman O. Development of carbon nanotubebased biosensors. International Journal of Nano and Biomaterials. 2016;6(2):83-109

31. Kang X, Wang J, Wu H, Aksay IA, Liu J, Lin Y. Glucose oxidasegraphene-chitosan modified electrode for direct electrochemistry and glucose sensing. Biosensors and Bioelectronics. 2009;25(4):901-5.

32. Palanisamy S, Cheemalapati S, Chen S-M. Amperometric glucose biosensor based on glucose oxidase dispersed in multiwalled carbon nanotubes/graphene oxide hybrid biocomposite. Materials Science and Engineering: C. 2014;34:207-13.

33. Hashemi SA, Mousavi SM, Bahrani S, Ramakrishna S. Integrated polyaniline with graphene oxide-iron tungsten nitride nanoflakes as ultrasensitive electrochemical sensor for precise detection of 4 
nitrophenol within aquatic media. Journal of Electroanalytical Chemistry. 2020:114406.

34. Hwa K-Y, Subramani B. Synthesis of zinc oxide nanoparticles on graphene-carbon nanotube hybrid for glucose biosensor applications. Biosensors and Bioelectronics. 2014;62:127-33

35. O'regan B, Grätzel M. A low-cost, high-efficiency solar cell based on dye-sensitized colloidal $\mathrm{TiO} 2$ films. nature. 1991;353(6346):737-40.

36. FACCIO N. Relatório de Campo das Áreas dos Sítios Arqueológicos Turvos. FCT/UNESP. 2011.

37. Mani V, Devadas B, Chen S-M. Direct electrochemistry of glucose oxidase at electrochemically reduced graphene oxide-multiwalled carbon nanotubes hybrid material modified electrode for glucose biosensor. Biosensors and Bioelectronics. 2013;41:309-15.

38. Dong X, Chen J, Ma Y, Wang J, Chan-Park MB, Liu X, et al. Superhydrophobic and superoleophilic hybrid foam of graphene and carbon nanotube for selective removal of oils or organic solvents from the surface of water. Chemical communications. 2012;48(86):10660-2

39. Huang J, Dai Y, Gu C, Sun Y, Liu J. Preparation of porous flowerlike $\mathrm{CuO} / \mathrm{ZnO}$ nanostructures and analysis of their gas-sensing property. Journal of alloys and compounds. 2013;575:115-22.

40. Wang J, Zhao Y, Ma F-X, Wang K, Wang F-B, Xia X-H. Synthesis of a hydrophilic poly-L-lysine/graphene hybrid through multiple non-covalent interactions for biosensors. Journal of materials Chemistry B. 2013;1(10):1406-13.

41. Vilian AE, Chen S-M, Ali MA, Al-Hemaid FM. Direct electrochemistry of glucose oxidase immobilized on $\mathrm{ZrO} 2$ nanoparticles-decorated reduced graphene oxide sheets for a glucose biosensor. RSC Advances. 2014;4(57):30358-67.

42. Wang R, Sun J, Gao L, Xu C, Zhang J. Fibrous nanocomposites of carbon nanotubes and graphene-oxide with synergetic mechanical and actuative performance. Chemical Communications. 2011;47(30):8650-2

43. Ma J, Zhou L, Li C, Yang J, Meng T, Zhou H, et al. Surfactantfree synthesis of graphene-functionalized carbon nanotube film as a catalytic counter electrode in dye-sensitized solar cells. Journal of Power Sources. 2014;247:999-1004.

44. Li S, Luo Y, Lv W, Yu W, Wu S, Hou P, et al. Vertically Aligned Carbon Nanotubes Grown on Graphene Paper as Electrodes in Lithium-Ion Batteries and Dye-Sensitized Solar Cells. Advanced Energy Materials. 2011;1(4):486-90.

45. Yen M-Y, Hsiao M-C, Liao S-H, Liu P-I, Tsai H-M, Ma C-CM, et al. Preparation of graphene/multi-walled carbon nanotube hybrid and its use as photoanodes of dye-sensitized solar cells. Carbon. 2011;49(11):3597-606.

46. Yuan D, Chen S, Yuan R, Zhang J, Liu X. An ECL sensor for dopamine using reduced graphene oxide/multiwall carbon nanotubes/gold nanoparticles. Sensors and Actuators B: Chemical. 2014;191:415-20.

47. Liu M, Li G, Chen X. One-pot controlled synthesis of spongelike CuInS2 microspheres for efficient counter electrode with graphene assistance in dye-sensitized solar cells. ACS applied materials \& interfaces. 2014;6(4):2604-10

48. Yang N, Zhai J, Wang D, Chen Y, Jiang L. Two-dimensional graphene bridges enhanced photoinduced charge transport in dyesensitized solar cells. ACS nano. 2010;4(2):887-94.

49. Yang N, Zhang Y, Halpert JE, Zhai J, Wang D, Jiang L. GranumLike Stacking Structures with TiO2-Graphene Nanosheets for Improving Photo-electric Conversion. Small. 2012;8(11):1762-70.

50. Gao H, Xiao F, Ching CB, Duan H. One-step electrochemical synthesis of $\mathrm{PtNi}$ nanoparticle-graphene nanocomposites for nonenzymatic amperometric glucose detection. ACS applied materials \& interfaces. 2011;3(8):3049-57.

51. Zhu G, Pan L, Lu T, Xu T, Sun Z. Electrophoretic deposition of reduced graphene-carbon nanotubes composite films as counter electrodes of dye-sensitized solar cells. Journal of Materials Chemistry. 2011;21(38):14869-75

52. Zheng H, Neo CY, Ouyang J. Highly efficient iodide/triiodide dyesensitized solar cells with gel-coated reduce graphene oxide/single-walled carbon nanotube composites as the counter electrode exhibiting an open-circuit voltage of $0.90 \mathrm{~V}$. ACS applied materials \& interfaces. 2013;5(14):6657-64.
53. Ahmad I, Khan U, Gun'ko YK. Graphene, carbon nanotube and ionic liquid mixtures: towards new quasi-solid state electrolytes for dye sensitised solar cells. Journal of Materials Chemistry. 2011;21(42):16990-6

54. Hu Y, Li X, Wang J, Li R, Sun X. Free-standing graphene-carbon nanotube hybrid papers used as current collector and binder free anodes for lithium ion batteries. Journal of power sources. 2013;237:41-6.

55. Yang Z-Y, Zhao Y-F, Xiao Q-Q, Zhang Y-X, Jing L, Yan Y-M, et al. Controllable growth of CNTs on graphene as high-performance electrode material for supercapacitors. ACS applied materials \& interfaces. 2014;6(11):8497-504.

56. Ahmad I, Yazdani B, Zhu Y. Recent advances on carbon nanotubes and graphene reinforced ceramics nanocomposites. Nanomaterials. 2015;5(1):90-114.

57. Evans AG. Perspective on the development of high-toughness ceramics. Journal of the American Ceramic society. 1990;73(2):187-206

58. Mousavi S, Zarei M, Hashemi S. Polydopamine for biomedical application and drug delivery system. Med Chem (Los Angeles) 2018;8:218-29.

59. Mousavi SM, Low FW, Hashemi SA, Samsudin NA, Shakeri M, Yusoff Y, et al. Development of hydrophobic reduced graphene oxide as a new efficient approach for photochemotherapy. RSC Advances. 2020;10(22):12851-63 